\title{
Placental infarction and intrauterine growth restriction following SARS-CoV-2 infection
}

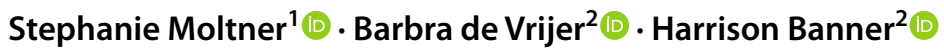

Received: 20 July 2021 / Accepted: 2 August 2021 / Published online: 18 August 2021

(c) The Author(s), under exclusive licence to Springer-Verlag GmbH Germany, part of Springer Nature 2021

Keywords SARS-CoV-2 Fetal Growth Restriction · Placenta $\cdot$ COVID-19

A previously healthy 29 -year-old G1 was admitted to the Intensive Care Unit at $29+5$ with severe thrombocytopenia and respiratory compromise resulting from SARS$\mathrm{CoV}-2$ infection. Ultrasound showed fetal growth at the 14th percentile with normal fluid and Dopplers.

At $32+4$, ultrasound demonstrated an almost complete growth arrest, with less than $100 \mathrm{~g}$ of growth in 3 weeks, measurements at the 6th percentile, and absent end-diastolic

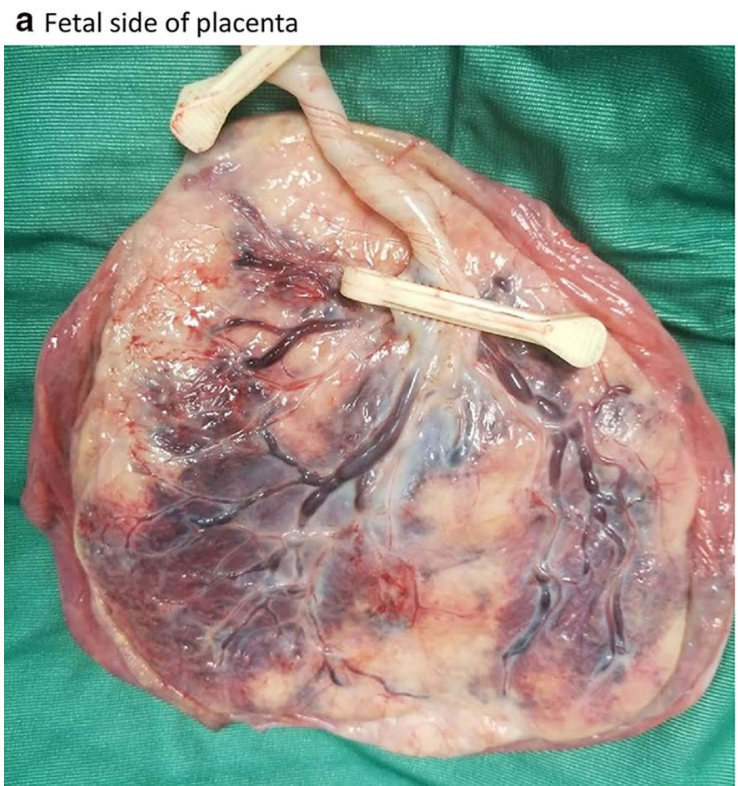

velocity (AEDV) in the umbilical artery. The patient was admitted for corticosteroids and fetal monitoring. Labour was induced at 34 weeks for AEDV and oligohydramnios and resulted in a vaginal birth of a male infant weighing $1559 \mathrm{~g}$ (4th percentile).

The placenta appeared grossly abnormal (Fig. 1) with $60 \%$ of the fetal surface demonstrating evidence of infarction and fetal vascular malperfusion. This case is a dramatic

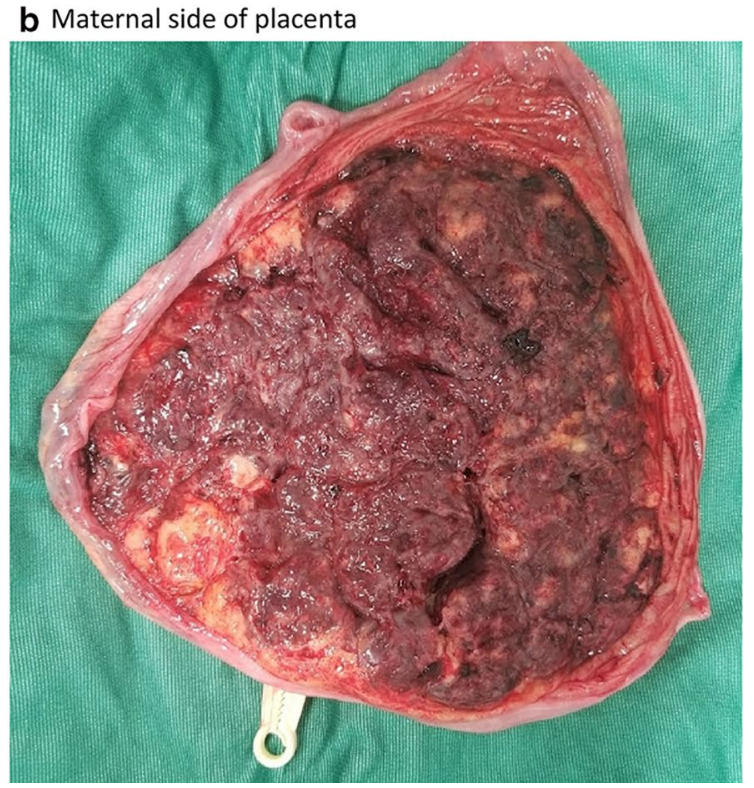

Fig. 1 Placenta demonstrating gross infarction following maternal SARS-CoV-2 infection

Harrison Banner

harrison.banner@lhsc.on.ca

1 Department of Obstetrics and Gynaecology, Schulich School of Medicine and Dentistry, Western University, London Health Sciences Centre, London, ON, Canada
2 Division of Maternal Fetal Medicine, Department of Obstetrics and Gynaecology, Schulich School of Medicine and Dentistry, Western University, London Health Sciences Centre, 800 Commissioners Road East, B2-412, London, ON N6A 5W9, Canada 
presentation of arrested fetal growth related to placental vascular pathology. Fetal growth and well-being should be monitored following SARS-CoV-2 infection.

Author contributions SM: manuscript writing/editing, BV: manuscript writing/editing, HB: manuscript writing/editing.

\section{Declarations}

Conflict of interest The authors have no conflicts of interest to declare.

Publisher's Note Springer Nature remains neutral with regard to jurisdictional claims in published maps and institutional affiliations. 